\title{
Arterialização seletiva da veia interventricular anterior: opção de revascularização miocárdica
}

Luiz Fernando KUBRUSLY *, Márcio ESCORSIN *, Antonio Carlos ZAVELINSKI *, Paulo Henrique STAHLKE *, Sérgio SAVYTZKY *, Denise Bermudez KUBRUSLY *.

KUBRUSLY, L. F.; ESCORSIN, M.; ZAVELINSKI, A. C.; STAHLKE, P. H.; SAVYTZKY, S.; KUBRUSLY, D. B. - Arterializaçāo seletiva da veia interventricular anterior: opçăo de revascularizaçăo miocárdica.

Rev. Bras. Cir. Cardiovasc. 7(4):283-286, 1992.

RESUMO: O objetivo deste trabalho é referir dois casos cirúrgicos, onde, após ressecção de aneurisma de ventrículo esquerdo, a revascularizaçāo do miocárdio através da artéria interventricular anterior (AIA) nāo foi possivel. Utilizando-se a artéria torácica interna (ATI) esquerda, optou-se pela anatomose com a veia interventricular anterior (VIA) com ligadura próxima, para estabelecimento do fluxo retrógrado e evitar a formação de fístula arteriovenosa. Não houve mortalidade e os resultados a curto prazo são encorajadores. Testes de perfusāo miocárdica com radioisótopos no pré e no pós-operatório, assim como angiografia do sistema coronário e da artéria torácica interna esquerda pré e pós-operatória são apresentados e discutidos. O objetivo da cirurgia de aneurisma ventricular é a recomposição geométrica do ventrículo esquerdo e revascularização de possíveis áreas isquêmicas. $\mathrm{Na}$ impossibilidade de revascularizaçāo direta da artéria interventricular anterior, a técnica citada demonstra ser facilmente exequível e, a curto prazo, com bons resultados.

DESCRITORES: miocárdio, revascularizaçāo, cirurgia.

\section{INTRODUÇÃO}

A possibilidade de perfusāo retrógrada através das veias do coração é descrita desde 1898 por PRATT ' . Este autor manteve um coração de gato por várias horas com perfusão através do seio coronário.

Outros trabalhos experimentais se sucederam, até que as primeiras tentativas clínicas de arterializaçāo do seio coronário foram descritas por ROBERTS et alii ${ }^{10}(1943)$ e BECK ${ }^{1}$ (1948). Pelo ano de 1954, Beck já havia descrito 186 casos das suas técnicas, (Beck 1 e 2), porém consistentemente, pelo menos para os nossos dias, com mortalidade e morbidade inaceitáveis. Complicaçōes, como formaçāo de fístula AV, hemorragia e edema mio- cárdico eram muito freqüentes, mesmo na operação de BECK ${ }^{2}$.

Mais recentemente MOLL et alii ${ }^{8}$ reportaram melhores resultados, porém, com o advento da anastomose aorto-artéria coronária, a operação de BECK 2 foi abandonada.

Várias publicações com respeito à proteção miocárdica retrógrada durante a cirurgia cardíaca vêm fortalecer nossos conhecimentos de que pode haver nutrição miocárdica via seio coronário, pelo sistema venoso do coração; a injeção de solução cardioplégica através do seio coronário em poucos segundos pode determinar a parada cardíaca.

Outros trabalhos de arterialização seletiva de

Trabalho realizado no Instituto do Coração de Curitiba, PR, Brasil.

Apresentado ao $19^{\circ}$ Congresso Nacional de Cirurgia Cardíaca, Sáo paulo, SP, 7 a 9 de maio, 1992.

* Do Instituto do Coração de Curitiba - Hospital Santa Cruz.

Endereço para separatas: Luiz Fernando Kubrusly. Rua Carneiro Lobo, 720 80240. Curitiba, PR, Brasil. 
KUBRUSLY, L. F.; ESCORSIN, M.; ZAVELINSKI, A. C.; STAHLKE, P. H.; ZAVYTZKY, S.; KUBRUSLY, D. B. - Arterialização seletiva da veia interventricular anterior: opção de revascularizaçăo miocárdica. Rev. Bras. Cir. Cardiovasc. 7(4):283-286, 1992.

veias coronárias ${ }^{2,5,6}$ foram publicados e demonstram, através de estudos com microesferas, que a perfusāo miocárdica ocorre principalmente quando há fechamento completo da artéria correspondente.

O objetivo deste trabalho é referir dois casos de ressecção de aneurisma do ventrículo esquerdo com posterior reconstruçāo geométrica e na impossibilidade de revascularizaçāo da artéria interventricular anterior, optou-se pela anastomose com a veia correspondente.

\section{CASUÍSTICA E MÉTODOS}

\section{Relato dos Casos}

Caso 1: O. C., 55 anos, sexo masculino, apresentou quadro de infarto agudo do miocárdio (IAM) em 16.07.89. Tratado clinicamente, apresentou-se, dois anos após, com quadro de insuficiência cardíaca. A cineangiocoronariografia revelou aneurisma de parede anterior do ventrículo esquerdo (VE)
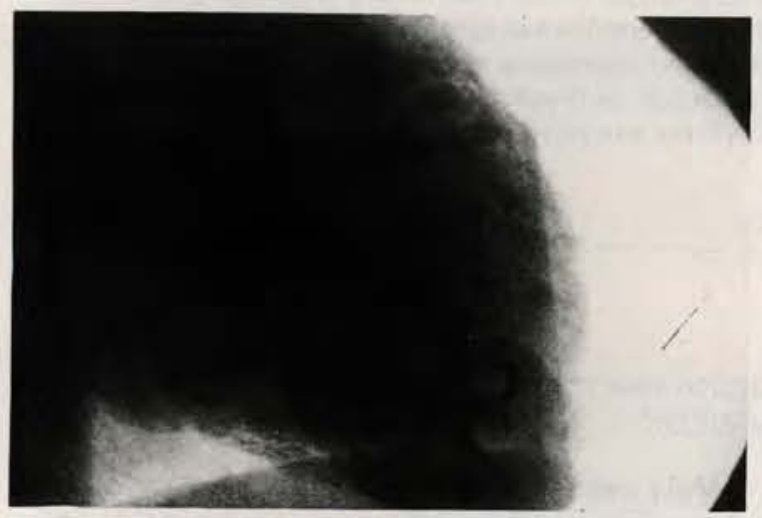

Fig. 1 - Ventriculografia em OAD pré-operatória.
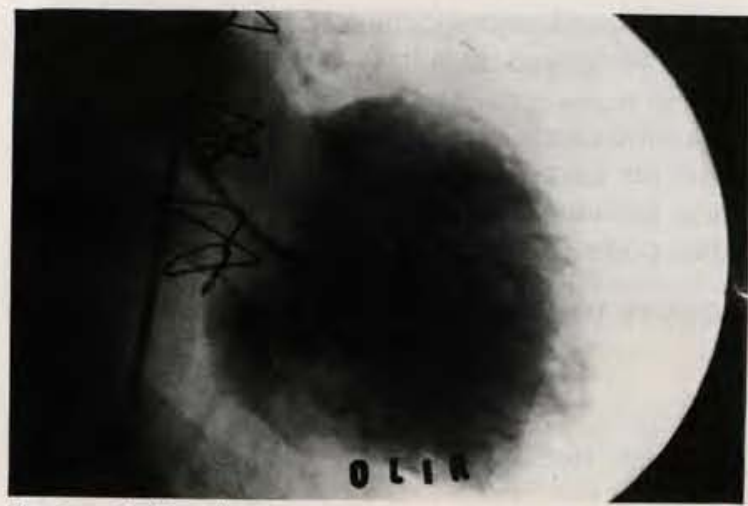

Fig. 2 - Cintilografia.
(Figura 1), lesão crítica no $1 / 3$ médio da coronária direita (CD) e obstrução total da artéria interventricular anterior sem sinais de recanalização. Tanto - ecocardiograma como a cintilografia miocárdica (Figura 2) revelaram parede septal hipocinética (com tecido viável) e isquemia de parede diafragmática. Indicado tratamento cirúrgico para aneurismectomia e revascularizaçāo do miocárdio.

Instalada a circulaçăo extracorpórea (CEC), infusão de soluçāo cardioplégica sangüínea, ressecçāo do aneurisma, reconstruçāo do ventrículo; passouse, então, a revascularização do miocárdio através da artéria coronária direita com veia safena. Após a exploração da AlA em vários pontos, concluiu-se da impossibilidade de anastomose nesta artéria. Optou-se, entāo, pela anastomose na veia interventricular anterior utilizando-se a artéria torácica interna esquerda com enxerto. A veia foi ligada cranialmente à anastomose, para evitar formaçāo de fístula arteriovenosa.

Não houve dificuldades após a saída de perfusão. A evolução pós-operatória foi excelente, com índice cardíaco passando de $1,61 / \mathrm{min}^{2}$ para 2,6 $1 / \mathrm{min} / \mathrm{m}^{2}$; alta hospitalar no $7^{\circ}$ dia de P.O. A ventriculografia pós-operatória (Figura 3) mostrou meIhora da fração de ejeção ( $40 \%$ para $50 \%$ ) com reduçāo do volume sistólico final do VE.

A coronariografia evidenciou a artéria torácica interna esquerda incluindo o sistema venoso anterior (Figura 4), com pequeno escape para o seio venoso. A cintilografia miocárdica feita no $30^{\circ}$ dia de P.O. mostrou melhora na captaçāo do radioisótopo em regiāo anterior (Figura 2 ), confirmando um aumento na perfusāo miocárdica na regiāo ântero-septal.

Caso 2: A. Z., 62 anos, com história de infarto agudo do miocárdio de parede anterior, há 30 dias, tratado com streptokinase, evoluindo com teste de esforço positivo.

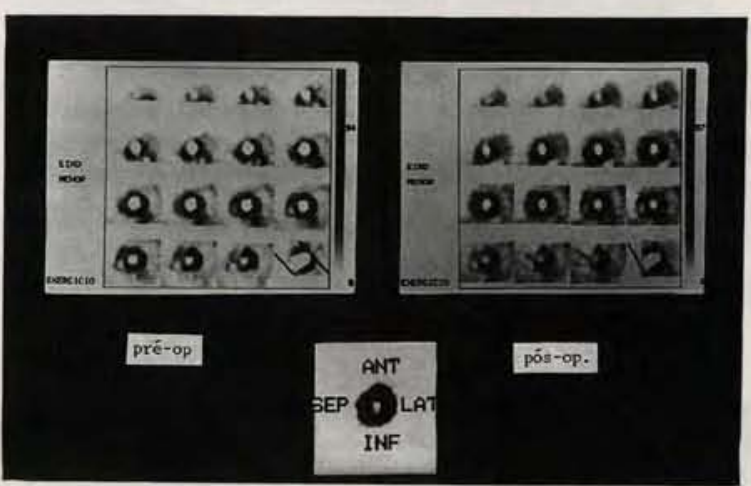

Fig. 3 - Ventriculografia em OAD pós-operatória. 

da veia interventricular anterior: opção de revascularizaçāo miocárdica. Rev. Bras. Cir. Cardiovasc. 7(4):283-286, 1992.

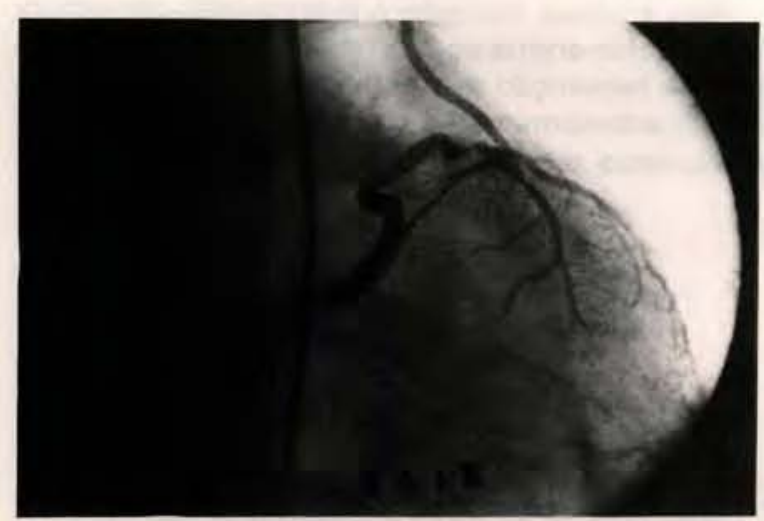

Fig. 4 - Artéria mamária interna anastomosada à veia descendente anterior; perfundindo o septo com circulação colateral para coronária direita; observe-se o escape para o seio coronário (vide texto).

Realizada cintilografia miocárdica que mostrou isquemia de várias áreas com tecido viável em parede anterior.

No cateterismo apresentava lesōes críticas em coronária direita, $1^{\circ}$ e $2^{\circ}$ ramos marginais da circunflexa e lesão total da artéria interventricular anterior com recanalizaçāo discreta. A ventriculografia mostrava pequeno aneurisma de ponta de VE.

Indicado tratamento cirúrgico. Após instalação da circulaçăo extracorpórea e infusāo de cardioplegia sangüínea, foi ressecado o aneurisma com reconstrução geométrica, seguido de revascularização do miocárdio com quatro pontes de veia safena, para C. D., $1^{a}$ e $2^{a}$ marginais e para AIA, esta última de $1 \mathrm{~mm}$ de diâmetro com mau leito distal. Terminadas pontes, o paciente foi retirado de CEC, com $5 \mathrm{mc} / \mathrm{gr} / \mathrm{kg} / \mathrm{min}$ de dopamina e levado à UTI. Com duas horas de pós-operaçăo, apresentou fibrilaçăo ventricular. Reanimado, foi levado ao centro cirúrgico; reinstalada a a $\mathrm{CEC}$, quando constatou-se oclusāo do enxerto para a AlA. Dissecada a artéria torácica interna esquerda e anastomosada à veia interventricular anterior. Houve grande dificuldade na saida da CEC, sendo necessária a colocaçāo de balāo intra-aórtico de contrapulsaçāo e bypass de ventrículo esquerdo com a bomba "Biomedicus". O suporte circulatório permaneceu por 72 horas.

No $13^{2}$ dia de pós-operatório, desenvolveu quadro de abdome agudo. Explorado cirurgicamente, evidenciou-se colecistite gangrenada sem cálculos, por provável baixo débito. Apresenta-se atualmente no $25^{\circ}$ dia de pós-operatório com excelente evoluçāo.

\section{COMENTÁRIOS}

A arterialização seletiva de veias do coração permite oferta imediata de sangue oxigenado para o miocárdio isquêmico.

O procedimento deste tipo realizado no passado 1.10 , seguiu-se de altas taxas de morbidade e mortalidade, até que deixou de ser utilizado, em razão do aparecimento da revascularização direta aorto-coronária.

No entanto, vários autores ${ }^{2,4.5}$ vêm demonstrando, com certa contradiçāo, variável "quantidade" de perfusāo miocárdica com tal procedimento, sendo reservado, portanto, para casos de doença aterosclerótica severa das artérias coronárias onde a revascularizaçāo padrāo nāo é factível.

Nas ressecçōes de aneurisma com reconstruçāo geométrica do ventrículo esquerdo, a revascularizaçāo da artéria interventricular anterior tem sido recomendada ${ }^{3}$ para nutrição de artérias septais e algumas artérias diagonais, e melhora, com isso, da funçāo ventricular.

$\mathrm{Na}$ maioria dos casos, no entanto, após a ressecçāo do aneurisma de VE, a porção proximal da artéria encontra-se altamente comprometida com processo aterosclerótico e, portanto, tornando a anastomose impraticável. Recomendamos, nestes casos, a utilização da veia com ligadura, para evitar formaçāo de fístula arteriovenosa. Outro aspecto importante a acrescentar é que a isquemia miocárdica tem papel fundamental na gênese de arritmias ventriculares graves ${ }^{7}$. Em pacientes com aneurisma de $\mathrm{VE}$ e isquemia confirmada pela cintilografia, com a artéria interventricular anterior sem possibilidades de revascularizaçāo, esta técnica se mostra como uma boa opçāo.

Vemos, no caso de número 1, que a artéria torácica interna foi capaz de encher a veia interventricular anterior, embora ainda com pequeno escape para o seio coronário. Inclusive as veias septais se enchem de forma completa, assim como o sistema venoso coronário direito.

A técnica aqui descrita e não encontrada na literatura, é a utilizaçāo de enxerto de artéria torácica interna (fluxo mais baixo que a veia safena) para a veia interventricular anterior, com sua ligadura cefálica em caso de ressecçāo de aneurisma de ventrículo esquerdo. Recomendamos, ainda, a ligadura de pequenas veias que comunicam a veia interventricular anterior ao sistema venoso coronário direito, o que facilitaria a entrada de sangue no sistema das septais e diagonais. Muitas vezes, isto ocorre automaticamente, quando ressecamos o aneurisma, estirpando todo tipo de conexāo vascular que possa existir. 
KUBRUSLY, L. F.; ESCORSIN, M.; ZAVELINSKI, A. C.; STAHLKE, P. H.; ZAVYTZKY, S.; KUBRUSLY, D. B. - Arterialização seletiva da veia interventricular anterior: opção de revascularizaçāo miocárdica. Rev. Bras. Cir. Cardiovasc. 7(4):283-286, 1992.

No segundo paciente, utilizamos esta técnica como já fora descrita anteriormente ${ }^{2,6}$ para casos onde a doença aterosclerótica coronária é difusa, principalmente em reoperaçōes, impossibilitando uma boa anastomose.
Nāo é nossa intensão a substituição da técnica original aorto-artéria coronária, porém indicamos em casos de ressecçāo de aneurisma de VE (ainda nāo descrito anteriormente) e processos ateroscleróticos coronarianos difusos e severos.

\section{RBCCV 44205-191}

KUBRUSLY, L. F.; ESCORSIN, M.; ZAVELINSKI, A. C.; STAHLKE, P. H.; SAVYTZKY, S.; KUBRUSLY, D. B. - Selective arterialization of the anterior interventricular vein: a choice for myocardial revascularization. Rev. Bras. Cir. Cardiovasc., 7(4):283-286, 1992.

ABSTRACT: The purpose of this study is to report two surgical cases, whereby after the repair of the left ventricular aneurysm, the myocardial revascularization of the interventricular anterior artery (IAA) was not possible. Using the left internal thoracic artery it was decided to perform anastomosis with the interventricular anterior (IAA) and its cephalad ligation, in order to obtain a retrograde flow and to avoid an arteriovenous fistula formation. There was no mortality and the results are optimistic in a short follow-up of these two patients. Myocardial perfusion tests and pre and post-operative angiography of the coronary system and the left internal thoracic artery are assessed and discussed. The geometrical reconstruction of the left ventricle and the revascularization of the ischemic areas play major role in the repair of postinfarction ventricular aneurysm. When it is not possible to access diretly the revascularization of the IAV this technique is safely and easily perfomed and shows satisfactory results in a short period of time.

DESCRIPTORS: myocardial revascularization, surgery.

\section{REFERÊNCIAS BIBLIOGRÁFICAS}

1 BECK, C. S. - Revascularization of the heart. Ann. Surg., 128: 854-860, 1948.

2 CHIU, C. J. \& MULDER, D. S. - Selective arterialization of coronary veins for diffuse coronary occlusion: an experimental evaluation. J. Thorac. Cardiovasc. Surg., 70: 177-182, 1975.

3 COOLEY, D. A. \& REUL, J. G. - Techniques in cardiac surgery. In: Revascularization of the ischemic myocardial. 2 ed., Washington, W. B. Saunders, 1984. p. 221-258.

ECKSTEIN, R. W.; HORIBERGER, J. C.; SANO, T. Acute effects of elevation of coronary sinus pressure. Circulation, 7: 422-428, 1953.

HOCHBERG, M. S. - Hemodynamic evaluation of selective arterialization of the coronary venous system: an experimental study of myocardial perfusion using radioactive microspheres. J. Thorac. Cardiovasc. Surg., 74: 774-783, 1977.
6 HOCHBERG, M. S.; ROBERTS, W. C.; MORROW, A. G. - Selective arterialization of the coronary venous system: encouraging long-term flow evaluation utilizing radioactive microspheres. J. Thorac. Cardiovasc. Surg., 77: 1-12, 1979.

7 KANNEL, W. B.; CUPPLES, A. L.; D' AGOSTINO, R. B. - Sudden death risk in overt coronary heart disease: the Framingham study. Am. Heart J., 113: 799 $805,1987$.

8 MOLL, J. W.; DZIATKWIAK, A.; RYBINSKI, K. Arterialisierunj des simus coronarius: Indikationen, Techink Ergebnisse. Thoraxchirurgie, 21: 295-298, 1973.

9 PRATT, F. H. - Nutrition of the heart through the vessels of Thebesius and coronary veins. Am. J. Physiol., 1: $86-90,1898$

10 ROBERTS, J. T.; BROWNE, H. S.; ROBERTS, G. Nourishment of the myocardium by way of the coronary veins. Fed. Proc., 2: 90-95, 1943. 